ORIGINAL ARTICLE

\title{
Percutaneous Endoscopic Gastrostomy Reduces Aspiration Pneumonia Rate in Stroke Patients with Enteral Feeding in Convalescent Rehabilitation Wards
}

\author{
Yasunori Ikenaga, MD, PhD ${ }^{\text {a,b }}$ Tadayuki Kusunoki, DS ${ }^{\mathrm{b}}$ and Hiromi Yamaguchi, MS, PhD ${ }^{\text {a }}$
}

\begin{abstract}
Objectives: The effect of percutaneous endoscopic gastrostomy (PEG) on the prevention of aspiration pneumonia and improvements in activities of daily living (ADL) for enteral feedingdependent stroke patients is unclear. We sought to clarify differences in the rates of aspiration pneumonia and ADL improvement between stroke patients receiving PEG and those receiving nasogastric tube feeding (NGT) in convalescent rehabilitation wards. Methods: We assessed 10 years of data from the Kaga Regional Cooperation Clinical Pathway for Stroke, which covers patients in the southern district of Ishikawa Prefecture of Japan. Logistic regression analysis with propensity score adjustment was used to examine how the enteral feeding method affected aspiration pneumonia rates. Linear regression analysis, adjusted by propensity scores, was also performed to ascertain the effect of the enteral feeding method on ADL improvement. Results: Overall, 47 patients with PEG and 49 patients with NGT were analyzed. The incidence of aspiration pneumonia was 4.67 times higher in the NGT group than in the PEG group in the propensity score-adjusted logistic regression analysis (odds ratio 4.67, 95\% confidence interval 1.30-16.67, $\mathrm{P}=0.02$ ). The enteral feeding method had no significant effect on ADL improvement in the propensity score-adjusted linear regression analysis. Conclusions: In convalescent rehabilitation wards, aspiration pneumonia was more likely to occur in stroke patients with NGT than in those with PEG; however, the enteral feeding method did not affect ADL improvement. These results provide a basis for determining the appropriate enteral feeding method for stroke patients who cannot take adequate nutrition orally during convalescence/rehabilitation.
\end{abstract}

Key Words: aspiration pneumonia; convalescent rehabilitation ward; nasogastric tube feeding; percutaneous endoscopic gastrostomy; stroke

\section{INTRODUCTION}

Dysphagia is a major complication after stroke that affects $27 \%$ to $64 \%$ of stroke patients. ${ }^{1,2)}$ About half of these patients can take food orally within 2 weeks of stroke onset, but others have prolonged dysphagia. ${ }^{3,4)}$ Previous studies have reported that stroke patients with prolonged dysphagia tend to develop aspiration pneumonia and have a longer hospital stay, a greater risk of mortality, and a lower rate of home discharge than patients without prolonged dysphagia. ${ }^{4-6)}$
Percutaneous endoscopic gastrostomy (PEG) and nasogastric tube feeding (NGT) are widely applied for stroke patients who cannot take adequate nutrition orally. ${ }^{7,8)}$ Previous studies have reported that long-term NGT causes aspiration pneumonia because of the presence of the nasal tube in the pharynx. ${ }^{9-11)}$ Other reports have suggested that NGT may interfere with improvements in the ability to perform activities of daily living (ADL) because patients sometimes need to be restrained to prevent self-removal of the NGT tube. ${ }^{12,13)}$ However, a recent meta-analysis comparing PEG and NGT

Received: April 24, 2021, Accepted: July 21, 2021, Published online: August 7, 2021

a Department of Rehabilitation Medicine, Yawata Medical Center, Ishikawa, Japan

${ }^{\mathrm{b}}$ Council of Kaga Local Stroke Network, South Ishikawa, Japan

Correspondence: Yasunori Ikenaga, MD, PhD, Yawata Medical Center, Department of Rehabilitation Medicine, 12-7 Yawata, Komatsu

City, Ishikawa 923-8551, Japan, E-mail: ikenaga@katsuki-g.com

Copyright (C) 2021 The Japanese Association of Rehabilitation Medicine

This is an open-access article distributed under the terms of the Creative Commons Attribution Non-Commercial No

Derivatives (CC BY-NC-ND) 4.0 License. http://creativecommons.org/licenses/by-nc-nd/4.0/ 
failed to show a statistically significant difference in the rates of aspiration pneumonia. ${ }^{14}$ ) This result indicates that the preventive effect of PEG on aspiration pneumonia for enteral feeding-dependent stroke patients remains unclear. In terms of improvements in ADL, to the best of our knowledge, no previous study has reported that PEG is more effective than NGT in improving ADL in enteral feeding-dependent stroke patients. Consequently, there is still no evidence on whether PEG is more effective in preventing aspiration pneumonia and more advantageous in terms of improving ADL than NGT for enteral feeding-dependent stroke patients.

One reason for the absence of a significant difference between PEG and NGT in terms of the rate of aspiration pneumonia is the heterogeneity among the study patients who had various diseases such as stroke and head and neck cancer and were in the acute and chronic phases of disease. ${ }^{14-17)}$ As a result, it is necessary to investigate the difference in the rates of aspiration pneumonia between patients receiving PEG and NGT by focusing on a specific disease and phase (acute, convalescent, or chronic) to clarify whether PEG or NGT is the optimal enteral feeding method for stroke patients who cannot take adequate nutrition orally.

Early PEG placement within 2 weeks after stroke onset reportedly increases the mortality and complication rates, ${ }^{18)}$ whereas continuous use of NGT in the chronic phase reportedly causes complications such as nasal ulcers, sinusitis, and reflux esophagitis. ${ }^{12)}$ Although there have been reports comparing PEG and NGT in the acute and chronic phases, we found no reports in the literature comparing PEG and NGT in the convalescence/rehabilitation phase when intensive rehabilitation is provided. ${ }^{19)}$ Because post-stroke patients are expected to gain the maximum improvement in motor function and ADL during the convalescence/rehabilitation phase, it is important to avoid aspiration pneumonia and to select the best enteral feeding method.

This study aimed to clarify the optimal nutritional management of enteral feeding-dependent stroke patients in convalescent rehabilitation wards. To this end, we determined whether there were differences in the rate of aspiration pneumonia, self-removal of the enteral feeding tube, and ADL improvement between dysphagic stroke patients receiving PEG and those receiving NGT in the convalescence/rehabilitation phase. We also examined whether PEG is superior to NGT in terms of the rate of aspiration pneumonia and ADL improvement by using regression analysis with propensity score adjustment.

\section{MATERIALS AND METHODS}

\section{Study Design}

This study used a case-control design to compare dysphagic stroke patients with enteral feeding via PEG or via NGT at the time of admission to convalescent rehabilitation wards (CRWs) and who were still receiving the same enteral feeding (PEG or NGT) at the time of discharge from the CRWs. PEG was recommended to the patients and/or their families by the doctors in charge at the acute care hospitals according to the guideline proposed by ESPEN. ${ }^{20)}$ If a patient and/or their family rejected PEG, their enteral nutrition was continued with NGT. All patients entered the CRWs within 2 months of stroke onset. Patients who were transferred to acute care hospitals from CRWs for intensive care or who died in a CRW were excluded from this study. All the patients eligible for analysis had medical care for aspiration pneumonia in the CRWs. Patients who were dependent on enteral feeding for diseases other than stroke and patients who were dependent on enteral feeding before the onset of stroke were also excluded.

\section{Database}

Ten years of data (2010-2019) from the Kaga Regional Cooperation Clinical Pathway for Stroke (KRCCPS) were analyzed for this study. KRCCPS is used in the southern part of Ishikawa Prefecture in Japan, a region populated by about 1 million people, and has been implemented since 2009 in 19 acute care hospitals, 11 hospitals with CRWs, and 466 facilities for community-based rehabilitation. By the end of 2019, KRCCPS had been used for more than 5000 patients and was deeply integrated in the district. ${ }^{21,22)}$ Patients' demographics; stroke-related assessments; and medical information from physicians, nurses, physical therapists, occupational therapists, speech-language-hearing therapists, pharmacists, nutritionists, health-care workers, and dentists are recorded in the KRCCPS using Microsoft Excel worksheets. The information in KRCCPS is shared throughout acute care hospitals, CRWs, and community-based rehabilitation facilities. Informed consent for using the data in KRCCPS was obtained from all the patients or their key persons at the time of admission to acute care hospitals.

In the KRCCPS database, the type of stroke (cerebral infarction, cerebral hemorrhage, or subarachnoid hemorrhage), the location of the lesion (infratentorial or supratentorial), paralyzed side/ataxia, first-time or recurrent stroke, date of onset, and the date of CRW admission and discharge were recorded. The National Institutes of Health Stroke 
Scale (NIHSS) score ${ }^{23)}$ was recorded to reflect the severity of the stroke. The NIHSS score on the day of stroke onset was evaluated by the stroke treatment team belonging to the acute care hospital. Complications, such as aspiration pneumonia, self-removal of the enteral feeding tube, hypertension, diabetes mellitus (DM), impaired lipid metabolism, and atrial fibrillation were recorded. The diagnosis of aspiration pneumonia was made according to the guidelines of the Japanese Respiratory Society. ${ }^{24)}$ The physiatrists in the CRWs diagnosed aspiration pneumonia based on the results of the infiltrative findings of chest X-rays and/or chest computed tomography, and inflammatory findings of serum blood tests such as high levels of C-reactive protein and high white blood cell counts. Because all the patients in this study had severe swallowing dysfunction, infiltrative findings on chest imaging combined with inflammatory findings on serum blood tests could be considered a diagnosis of aspiration pneumonia. The Dysphagia Severity Scale (DSS) and Functional Oral Intake Scale (FOIS) scores were recorded to evaluate the severity of dysphagia. The DSS can be used to evaluate the patient's swallowing function because of acceptable levels of interclass reliability and validity established in previous studies. ${ }^{25-29)}$ The FOIS is a well-established and reliable scale that is used internationally to evaluate a patient's capability for oral intake. ${ }^{30-32)}$ Body mass index (BMI; kg/ $\left.\mathrm{m}^{2}\right)^{33)}$ was recorded on the dietitian's sheet. The Functional Independence Measure (FIM) was used to evaluate the level of ADL. The FIM is a well-established, reliable, and validated scale of ADL, with 13 items for motor function and 5 items for cognitive function. ${ }^{34,35)}$ The DSS, FOIS, BMI, and FIM were evaluated by trained multidisciplinary teams in the CRWs on the days of admission and discharge. All patients received indirect and/or direct swallowing training provided by speech-language-hearing therapists in the CRWs, and if possible, patients started eating selected food items.

\section{Variables and Measures}

Age; sex; initial or recurrent stroke; type of stroke; lesion location (supratentorial or infratentorial); and motor impairment features (right, left, bilateral, or ataxia) were compared between the groups. The NIHSS score was used to compare the severity of stroke between the groups. The incidence of aspiration pneumonia is reportedly higher in patients with $\mathrm{DM} ;{ }^{36,37)}$ therefore, we also compared the rates of DM between the two groups. Because a difference in the rate of aspiration pneumonia has been reported depending on the severity of dysphagia, ${ }^{38)}$ we compared the DSS and FOIS scores at admission to the CRWs between the two groups.
Furthermore, low nutritional status has been associated with the development of aspiration pneumonia; ${ }^{39,40)}$ as a result, we compared BMI at admission to the CRWs between the two groups. The FIM score at admission to the CRWs and the improvement in FIM score during hospitalization in the CRW were compared between the two groups. The outcomes of patients are better in wards managed by board-certified physiatrists than in wards managed by non-board-certified physiatrists ${ }^{41)}$ consequently, we compared the number of patients who were hospitalized in CRWs managed by boardcertified physiatrists between the groups. The improvement in FIM score and the increase in BMI during hospitalization in the CRWs were compared to determine whether there were differences in ADL improvement and changes in nutritional status depending on the type of enteral feeding. The duration of hospitalization in CRWs was also compared between the two groups.

\section{Data Analysis}

SPSS version 26.0 for Windows (IBM Corporation, Armonk, NY, USA) was used for statistical analysis. Demographic data were compared using the Mann-Whitney U-test or the chi-squared test. Because the aim of this study was to identify the effect of PEG on the prevention of aspiration pneumonia, we set the rate of aspiration pneumonia as the dependent factor and the type of enteral feeding (PEG or NGT) as the independent factor with propensity score adjustment. Logistic regression analysis was used to calculate the odds ratio of the rate of aspiration pneumonia during hospitalization in the CRWs. A linear regression analysis was performed with the increase in FIM motor score during hospitalization in CRWs as the dependent variable and the type of enteral feeding as the independent variable, adjusted by the propensity score, to examine whether the type of enteral feeding affected the FIM motor score gain. If no significant difference was obtained, stepwise linear regression analysis was used to identify the independent factors that affect FIM motor score gains.

\section{Ethical Considerations}

All data in the KRCCPS were anonymized and the patients were not identified. This study was approved by the Ethics Committee of Yawata Medical Center in Ishikawa, Japan. 


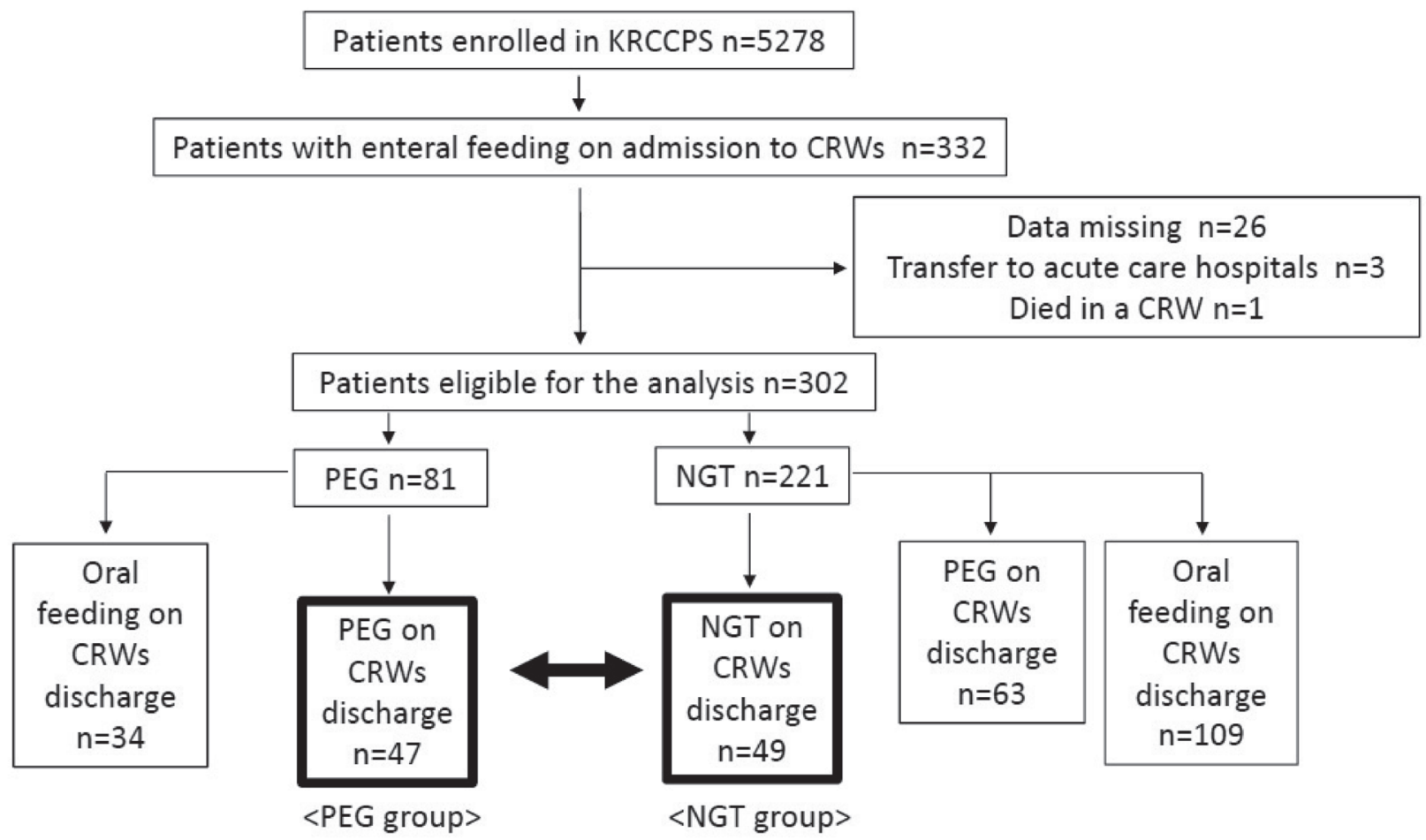

KRCCPS: Kaga Regional Cooperation Clinical Pathway for Stroke, CRW: convalescent rehabilitation ward, PEG: percutaneous endoscopic gastrostomy, NGT: nasogastric tube feeding

Fig. 1. Flowchart of patient selection criteria.

\section{RESULTS}

We screened the data of 5278 KRCCPS cases and found 332 patients who were enteral feeding-dependent at the time of admission to the CRWs. Twenty-six were excluded due to missing data. Four patients were excluded from this study: three were transferred to acute care hospitals from CRWs for intensive care and one patient died in a CRW. Overall, 143 patients were weaned from enteral feeding during their stay in the CRWs, and 63 patients were switched from NGT to PEG. Finally, 96 cases were eligible for inclusion in this study: 47 cases in the PEG group and 49 cases in the NGT group (Fig. 1).

There were no statistical differences in age, sex, stroke incidence, type of stroke, lesion location, rate of DM, or NIHSS scores at stroke onset between the PEG and the NGT group (Table 1). On admission to the CRWs, the PEG group had stayed in the acute care hospitals for about 12 days longer than the NGT group; however, the FIM cognitive score was lower in the NGT group (Table 2). During hospitalization in the CRWs, the rate of aspiration pneumonia was higher in the NGT group than in the PEG group. Furthermore, the rate of self-removal of the enteral feeding tube was $38.8 \%$ in the NGT group, whereas none of the patients in the PEG group removed their tube (Table 3). The FIM motor score at the time of discharge from the CRWs was higher in the PEG group (Table 4).

The preventive effect of PEG on aspiration pneumonia during hospitalization in CRWs was analyzed by logistic regression analysis with propensity score adjustment. The cstatistic was 0.85 , and the adjustment of the propensity score was deemed appropriate. The odds ratio for the incidence of aspiration pneumonia in the NGT group was 4.67, indicating that aspiration pneumonia was 4.67 times more likely in the NGT group than in the PEG group ( $\beta=1.54$, odds ratio 4.67 , $95 \%$ confidence interval $[\mathrm{CI}] 1.30-16.67, \mathrm{P}=0.02)$. The type of enteral feeding (PEG or NGT) did not have a statistically significant effect on the increase in FIM motor score at the time of discharge from the CRWs in the linear regression analysis adjusted by the propensity score $(\beta=-0.97,95 \% \mathrm{CI}$ -3.51 to $1.58, \mathrm{P}=0.45)$. We chose the number of days from the onset of stroke to admission to the CRWs, the FIM cognitive score at the time of admission to the CRWs, and the type of enteral feeding as independent factors for contributing to the 
Table 1. Characteristics of patients at the onset of stroke

\begin{tabular}{lccc}
\hline Characteristic & PEG group (n=47) & NGT group (n=49) & P values \\
\hline Age (years) & $77.94 \pm 10.13$ & $78.53 \pm 11.52$ & 0.61 \\
Sex, male/female & $26 / 21$ & $26 / 23$ & 0.84 \\
Stroke incidence, initial/recurrent & $30 / 17$ & $38 / 11$ & 0.18 \\
Type of stroke, & $26 / 17 / 4$ & $24 / 20 / 5$ & 0.82 \\
infarction/hemorrhage/subarachnoid hemorrhage & $38 / 9$ & $43 / 6$ & 0.41 \\
Lesion location, supratentorial/infratentorial & $6 / 41$ & $11 / 38$ & 0.29 \\
Diabetes mellitus, yes/no & $20.04 \pm 8.20$ & $19.07 \pm 6.99$ & 0.15 \\
NIHSS score & & \\
\hline
\end{tabular}

Data are presented as mean \pm standard deviation or $\mathrm{n}$.

Table 2. Characteristics of patients at the time of admission to convalescent rehabilitation wards

\begin{tabular}{lccc}
\hline Characteristic & PEG group $(\mathrm{n}=47)$ & NGT group $(\mathrm{n}=49)$ & P values \\
\hline Time from the onset of stroke & $47.87 \pm 8.09$ & $35.92 \pm 14.03$ & $<0.01^{* *}$ \\
to admission to CRW (days) & $17 / 14 / 15 / 1$ & $12 / 26 / 9 / 2$ & 0.10 \\
Paresis side or ataxia, right/left/bilateral/ataxia & $1.74 \pm 0.79$ & $1.51 \pm 0.68$ & 0.14 \\
DSS score & $1.40 \pm 0.54$ & $1.41 \pm 0.57$ & 0.94 \\
FOIS score & $13.98 \pm 3.24$ & $13.86 \pm 3.36$ & 0.71 \\
FIM motor score & $8.57 \pm 3.73$ & $7.43 \pm 4.29$ & $0.03^{*}$ \\
FIM cognitive score & $20.06 \pm 2.40$ & $20.67 \pm 3.06$ & 0.06 \\
BMI (kg/m ${ }^{2}$ ) & $38 / 9$ & $45 / 4$ & 0.14 \\
CRW managed by certified physiatrists, yes/no & & \\
\hline
\end{tabular}

Data are presented as the mean \pm standard deviation or $\mathrm{n}$.

$*$ Statistically significant, $\mathrm{P}<0.05$; ** $\mathrm{P}<0.01$.

Table 3. Characteristics of patients during hospitalization in convalescent rehabilitation wards

\begin{tabular}{lccc}
\hline Characteristic & PEG group $(\mathrm{n}=47)$ & NGT group $(\mathrm{n}=49)$ & P values \\
\hline Aspiration pneumonia, yes/no & $6 / 41$ & $18 / 31$ & $0.01^{*}$ \\
Self-removal of the enteral feeding tube, yes/no & $0 / 47$ & $19 / 30$ & $<0.01^{* *}$ \\
\hline
\end{tabular}

Data are presented as $n$.

*Statistically significant, $\mathrm{P}<0.05 ; * * \mathrm{P}<0.01$.

Table 4. Characteristics of patients at the time of discharge from convalescent rehabilitation wards

\begin{tabular}{lccc}
\hline Characteristic & PEG group $(\mathrm{n}=47)$ & NGT group $(\mathrm{n}=49)$ & P values \\
\hline FIM motor score & $16.87 \pm 6.62$ & $15.65 \pm 7.01$ & $0.02 *$ \\
FIM cognitive score & $10.49 \pm 5.30$ & $9.69 \pm 6.94$ & 0.07 \\
FIM motor score gain & $2.89 \pm 5.11$ & $2.02 \pm 4.89$ & 0.08 \\
FIM cognitive score gain & $1.91 \pm 3.37$ & $2.27 \pm 4.86$ & 0.52 \\
BMI $\left(\mathrm{kg} / \mathrm{m}^{2}\right)$ & $19.03 \pm 2.55$ & $19.36 \pm 3.04$ & 1.0 \\
BMI change $\left(\mathrm{kg} / \mathrm{m}^{2}\right)$ & $-1.03 \pm 2.59$ & $-1.31 \pm 2.24$ & 0.28 \\
Duration of CRW stay (days) & $113.74 \pm 50.42$ & $122.96 \pm 60.16$ & 0.42 \\
\hline
\end{tabular}

Data are presented as the mean \pm standard deviation.

*Statistically significant, $\mathrm{P}<0.05$. 
increase in FIM motor score at the time of discharge from the CRWs. Stepwise linear regression analysis rejected the time from the onset of stroke to admission to the CRWs and the type of enteral feeding, leaving only the FIM cognitive score at admission to the CRWs as a significant contributor to the increase in motor FIM score $(\beta=0.45,95 \%$ CI $0.22-0.69$, $\mathrm{P}<0.01)$

\section{DISCUSSION}

In this study, we found that aspiration pneumonia was 4.67 times more likely to develop in enteral feeding-dependent stroke patients in the CRWs managed with NGT than with PEG. Overall, $38.8 \%$ of patients in the NGT group selfremoved the feeding tube during hospitalization, whereas none in the PEG group did so. The type of enteral feeding (PEG or NGT) did not have any effect on the increase in FIM motor score at the time of discharge from the CRWs, but the cognitive FIM score at the time of admission to the CRWs was a statistically significant contributor to such gain. No previous report has clarified the effect of PEG on the prevention of aspiration pneumonia for enteral feeding-dependent stroke patients in the convalescence/rehabilitation phase or investigated whether the enteral feeding method affected ADL improvement.

A recent meta-analysis comparing PEG and NGT did not show a statistically significant difference in the rate of aspiration pneumonia. ${ }^{14)}$ One of the reasons for the absence of a statistically significant difference in the rate of aspiration pneumonia between those receiving PEG and NGT was heterogeneity arising from the inclusion of patients with various diseases, such as stroke and head and neck cancer, and those in the acute and chronic phases of the disease. ${ }^{14-17)}$ In the current study, we compared the effect of PEG and NGT only in patients with stroke who were in CRWs and who had severe dysphagia. We also selected patients who continued to receive the same method of enteral feeding (PEG or NGT) during their entire stay in CRWs. In other words, our study included statistically homogeneous patients, and this may have helped to clarify the effect of PEG, compared with that of NGT, in preventing aspiration pneumonia.

Chang et al. evaluated the severity of dysphagia based on video endoscopic findings and concluded that PEG was more effective than NGT in preventing aspiration pneumonia in patients with severe dysphagia who had marked secretions in their pharynx. ${ }^{17)}$ In our study, patients in both the PEG and NGT groups had severe dysphagia, and our findings indicated that PEG was more effective than NGT in prevent- ing aspiration pneumonia, which was in agreement with the findings of Chang et al.

Baeton et al. reported that patients in the NGT group were restrained three times more often than those in the PEG group to prevent self-removal of the enteral feeding tube. They also concluded that PEG should be chosen for longterm tube-feeding management because NGT causes more discomfort than PEG. ${ }^{12)}$ Allison et al. and Park et al. reported that more patients self-extracted the NGT than the PEG because the former caused discomfort of the nasopharynx. ${ }^{13,42)}$ In our study, self-removal of the enteral feeding tube did not occur in the PEG group, but it occurred in $38.8 \%$ of patients in the NGT group. This result clearly demonstrated that selfremoval of the enteral feeding tube was much more likely to occur in the NGT group than in the PEG group, which is consistent with the conclusions of Park et al. and Allison et al. Therefore, PEG is recommended when prolonged enteral feeding is required.

In this study, the increase in FIM motor score was higher in the PEG group, but when linear regression analysis adjusted by the propensity score was used to examine whether the type of enteral feeding affected the FIM motor score gain, there was no statistically significant difference. According to the stepwise linear regression analysis, which included the time from the onset of stroke to admission to the CRWs, the FIM cognitive score at the time of admission to the CRWs, and the enteral feeding method as independent variables, only the FIM cognitive score at admission to the CRWs contributed to the increase in FIM motor score. In previous studies that examined variables affecting ADL in stroke patients, cognitive function was shown to affect ADL improvement, ${ }^{43-45)}$ which was concordant with our result. Whether PEG has a beneficial effect on ADL improvement compared with NGT needs to be examined in a database with a larger number of cases.

This study had several limitations due to its design as a retrospective case-control study that analyzed data from multiple centers. First, medication use was not taken into consideration. It has been reported that angiotensin-converting enzyme inhibitors can prevent aspiration pneumonia, ${ }^{46)}$ but a recent analysis with a large number of cases did not support this finding. ${ }^{47)}$ Furthermore, it has been stated that cilostazol can prevent aspiration pneumonia, ${ }^{48)}$ but this was based on a small number of cases. Consequently, it remains unclear whether these drugs can in fact prevent aspiration pneumonia. Therefore, it is unlikely that differences in the medications used affected the rate of aspiration pneumonia in our study. Second, chronic obstructive pulmonary disease 
(COPD), a respiratory disease that is easily complicated by pneumonia, ${ }^{49,50)}$ was not considered. However, the diagnosis of COPD requires spirometery, ${ }^{51)}$ and the patients investigated in this study had severe disability, making it almost impossible to examine them using spirometry. Appropriate enteral feeding (PEG or NGT) for dysphagic stroke patients with COPD needs to be clarified by a prospective study recruiting patients with a diagnosis of COPD before the onset of stroke. Third, aspiration pneumonia was diagnosed clinically, and pneumonia types other than aspiration pneumonia may also have been included. However, the definition of aspiration pneumonia remains vague and unclear. ${ }^{52,53)}$ Because all the patients in this study had severe dysphagia, and the diagnosis of aspiration pneumonia was made according to the guidelines of the Japanese Respiratory Society, ${ }^{24)}$ pneumonia that occurred during hospitalization in CRWs in this study was likely to be aspiration pneumonia.

In conclusion, this study clarified the effect of PEG on the prevention of aspiration pneumonia in stroke patients requiring enteral feeding during the convalescence/rehabilitation phase. Our results provide a basis for determining the appropriate enteral feeding method for dysphagic stroke patients who undergo rehabilitation in CRWs.

\section{CONFLICTS OF INTEREST}

The authors declare that there are no conflicts of interest.

\section{REFERENCES}

1. Wolfe CD, Taub NA, Woodrow J, Richardson E, Warburton FG, Burney PG: Patterns of acute stroke care in three districts of southern England. J Epidemiol Community Health 1993;47:144-148. DOI:10.1136/ jech.47.2.144, PMID:8326273

2. Smithard D, Renwick D, Martin D, O'Neill P: Chest infection following acute stroke: does aspiration matter? Age Ageing 1993;22(suppl 3):P24. DOI:10.1093/ ageing/22.suppl_3.P24-a

3. Singh S, Hamdy S: Dysphagia in stroke patients. Dysphagia 1997;12:188-193.

4. Smithard DG, O’Neill PA, England RE, Park CL, Wyatt R, Martin DF, Morris J: The natural history of dysphagia following a stroke. Dysphagia 1997;12:188-193. DOI:10.1007/PL00009535, PMID:9294937
5. Mann G, Hankey GJ, Cameron D: Swallowing disorders following acute stroke: prevalence and diagnostic accuracy. Cerebrovasc Dis 2000;10:380-386. DOI:10.1159/000016094, PMID:10971024

6. Perry L: Eating and dietary intake in communicationimpaired stroke survivors: a cohort study from acute-stage hospital admission to 6 months poststroke. Clin Nutr 2004;23:1333-1343. DOI:10.1016/j. clnu.2004.04.009, PMID:15556255

7. Bath PM, Bath FJ, Smithard DG: Interventions for dysphagia in acute stroke. Cochrane Database Syst Rev 2000;CD000323. PMID:10796343

8. Geeganage C, Beavan J, Ellender S, Bath PM: Interventions for dysphagia and nutritional support in acute and subacute stroke. Cochrane Database Syst Rev 2012;10:CD000323. DOI:10.1002/14651858.CD000323. pub2, PMID:23076886

9. Fay DE, Poplausky M, Gruber M, Lance P: Long-term enteral feeding: a retrospective comparison of delivery via percutaneous endoscopic gastrostomy and nasoenteric tubes. Am J Gastroenterol 1991;86:1604-1609. PMID:1951237

10. Wicks C, Gimson A, Vlavianos P, Lombard M, Panos M, Macmathuna P, Tudor M, Andrews K, Westaby D: Assessment of the percutaneous endoscopic gastrostomy feeding tube as part of an integrated approach to enteral feeding. Gut 1992;33:613-616. DOI:10.1136/ gut.33.5.613, PMID:1612476

11. Dwolatzky T, Berezovski S, Friedmann R, Paz J, Clarfield AM, Stessman J, Hamburger R, Jaul E, Friedlander Y, Rosin A, Sonnenblick M: A prospective comparison of the use of nasogastric and percutaneous endoscopic gastrostomy tubes for long-term enteral feeding in older people. Clin Nutr 2001;20:535-540. DOI:10.1054/clnu.2001.0489, PMID:11884002

12. Baeten $C$, Hoefnagels J: Feeding via nasogastric tube or percutaneous endoscopic gastrostomy. A comparison. Scand J Gastroenterol 1992;27(supp194):95-98. DOI:10.3109/00365529209096035, PMID:1298056

13. Allison MC, Morris AJ, Park RH, Mills PR: Percutaneous endoscopic gastrostomy tube feeding may improve outcome of late rehabilitation following stroke. J R Soc Med 1992;85:147-149. PMID:1556716 
14. Gomes CA Jr, Andriolo RB, Bennett C, Lustosa SA, Matos D, Waisberg DR, Waisberg J: Percutaneous endoscopic gastrostomy versus nasogastric tube feeding for adults with swallowing disturbances. Cochrane Database Syst Rev 2015;5:CD008096. DOI:10.1002/14651858. CD008096.pub4, PMID:25997528

15. Jaafar MH, Mahadeva S, Morgan K, Tan MP: Percutaneous endoscopic gastrostomy versus nasogastric feeding in older individuals with non-stroke dysphagia: a systematic review. J Nutr Health Aging 2015;19:190197. DOI:10.1007/s12603-014-0527-z, PMID:25651445

16. Wang J, Liu M, Liu C, Ye Y, Huang G: Percutaneous endoscopic gastrostomy versus nasogastric tube feeding for patients with head and neck cancer: a systematic review. J Radiat Res (Tokyo) 2014;55:559-567. DOI:10.1093/jrr/rrt144, PMID:24453356

17. Chang WK, Huang HH, Lin HH, Tsai CL: Percutaneous endoscopic gastrostomy versus nasogastric tube feeding: oropharyngeal dysphagia increases risk for pneumonia requiring hospital admission. Nutrients 2019;11:2969. DOI:10.3390/nu11122969, PMID:31817381

18. Dennis MS, Lewis SC, Warlow C, FOOD Trial Collaboration: Effect of timing and method of enteral tube feeding for dysphagic stroke patients (FOOD): a multicentre randomised controlled trial. Lancet 2005;365:764-772. DOI:10.1016/S0140-6736(05)179835, PMID:15733717

19. Miyai I, Sonoda S, Nagai S, Takayama Y, Inoue Y, Kakehi A, Kurihara M, Ishikawa M: Results of new policies for inpatient rehabilitation coverage in Japan. Neurorehabil Neural Repair 2011;25:540-547. DOI:10.1177/1545968311402696, PMID:21451116

20. Löser C, Aschl G, Hébuterne X, Mathus-Vliegen EM, Muscaritoli M, Niv Y, Rollins H, Singer P, Skelly RH: ESPEN guidelines on artificial enteral nutrition - percutaneous endoscopic gastrostomy (PEG). Clin Nutr 2005;24:848-861. DOI:10.1016/j.clnu.2005.06.013, PMID:16261664

21. Ikenaga Y: Quantitative text analysis and comparison of the amounts of information contained in the Kaga regional cooperation clinical pathway for strokes and conventional medical information letters [in Japanese]. J Jpn Soc Clin Pathw 2018;2:103-110.

22. Ikenaga Y: Effect of integration of a regional cooperation clinical pathway for strokes into ID-Link for work efficiency of nurses [in Japanese]. J Jpn Soc Clin Pathw 2020;3:176-181.
23. Lyden P, Brott T, Tilley B, Welch KM, Mascha EJ, Levine S, Haley EC, Grotta J, Marler J, NINDS TPA Stroke Study Group: Improved reliability of the NIH Stroke Scale using video training. Stroke 1994;25:2220-2226. DOI:10.1161/01.STR.25.11.2220, PMID:7974549

24. Japanese Respiratory Society: Aspiration pneumonia. Respirology 2009;14(Suppl 2):S59-S64. DOI:10.1111/ j.1440-1843.2009.01578.x, PMID:19857224

25. Tohara H, Palmer JB, Reynolds K, Kuhlemeier KV, Palmer S: Dysphagia severity scale [in Japanese]. J Stomatol Soc Jpn 2003;70:242-248. DOI:10.5357/ koubyou.70.242, PMID:14733127

26. Nishimura K, Kagaya H, Shibata S, Onogi K, Inamoto Y, Ota K, Miki T, Tamura S, Saitoh E: Accuracy of Dysphagia Severity Scale rating without using videoendoscopic evaluation of swallowing [in Japanese]. Jpn J Compr Rehabil Sci 2015;6:124-128.

27. Kagaya H, Okada S, Shigeta R, Ogata N, Ota K, Shibata $\mathrm{S}$, Saitoh E: Dysphagia associated with unilateral vocal cord immobility after cardiovascular surgery. Am J Phys Med Rehabil 2011;90:901-907. DOI:10.1097/ PHM.0b013e31822deb32, PMID:21952214

28. Wakabayashi H, Sashika H, Matsushima M: Head lifting strength is associated with dysphagia and malnutrition in frail older adults. Geriatr Gerontol Int 2015;15:410-416. DOI:10.1111/ggi.12283, PMID:24690326

29. Ikenaga Y, Nakayama S, Taniguchi H, Ohori I, Komatsu N, Nishimura H, Katsuki Y: Factors predicting recovery of oral intake in stroke survivors with dysphagia in a convalescent rehabilitation ward. J Stroke Cerebrovasc Dis 2017;26:1013-1019. DOI:10.1016/j. jstrokecerebrovasdis.2016.12.005, PMID:28108097

30. Crary MA, Mann GD, Groher ME: Initial psychometric assessment of a functional oral intake scale for dysphagia in stroke patients. Arch Phys Med Rehabil 2005;86:1516-1520. DOI:10.1016/j.apmr.2004.11.049, PMID:16084801

31. Kushner DS, Johnson-Greene D, Cordero MK, Thomashaw SA, Rodriguez J: Swallowing outcomes and discharge destinations in acute stroke tube-feeding-dependent dysphagia patients treated with neuromuscular electrical stimulation during inpatient rehabilitation. Am J Phys Med Rehabil 2020;99:487-494. DOI:10.1097/ PHM.0000000000001353, PMID:31764228 
32. Matsuda Y, Karino M, Kanno T: Relationship between the functional oral intake scale (FOIS) and the selfefficacy scale among cancer patients: a cross-sectional study. Healthcare (Basel) 2020;8:269. DOI:10.3390/ healthcare8030269, PMID:32823778

33. WHO Expert Consultation: Appropriate body-mass index for Asian populations and its implications for policy and intervention strategies. Lancet 2004;363:157-163. DOI:10.1016/S0140-6736(03)15268-3, PMID:14726171

34. Granger CV, Cotter AC, Hamilton BB, Fiedler RC: Functional assessment scales: a study of persons after stroke. Arch Phys Med Rehabil 1993;74:133-138. PMID:8431095

35. Ottenbacher KJ, Hsu Y, Granger CV, Fiedler RC: The reliability of the functional independence measure: a quantitative review. Arch Phys Med Rehabil 1996;77:1226-1232. DOI:10.1016/S00039993(96)90184-7, PMID:8976303

36. van der Maarel-Wierink CD, Vanobbergen JN, Bronkhorst EM, Schols JM, de Baat C: Risk factors for aspiration pneumonia in frail older people: a systematic literature review. J Am Med Dir Assoc 2011;12:344-354. DOI:10.1016/j.jamda.2010.12.099, PMID:21450240

37. Ozer MN, Uzunlulu M, Oguz A, Kostek O, Akyer E, Takir M: The effect of sociodemographic and clinical features on mortality in patients with diagnosis of aspiration pneumonia. North Clin Istanb 2015;2:41-47. PMID:28058338

38. Wilmskoetter J, Herbert TL, Bonilha HS: Factors associated with gastrostomy tube removal in patients with dysphagia after stroke: a review of the literature. Nutr Clin Pract 2017;32:166-174. DOI:10.1177/0884533616661012, PMID:29927529

39. Maeda K, Akagi J: Muscle mass loss is a potential predictor of 90-day mortality in older adults with aspiration pneumonia. J Am Geriatr Soc 2017;65:e18-e22. DOI:10.1111/jgs.14543, PMID:27858956

40. Matsusaka K, Kawakami G, Kamekawa H, Momma H, Nagatomi R, Itoh J, Yamaya M: Pneumonia risks in bedridden patients receiving oral care and their screening tool: malnutrition and urinary tract infection-induced inflammation. Geriatr Gerontol Int 2018;18:714-722. DOI:10.1111/ggi.13236, PMID:29380508
41. Kinoshita S, Kakuda W, Momosaki R, Yamada N, Sugawara H, Watanabe S, Abo M: Clinical management provided by board-certificated physiatrists in early rehabilitation is a significant determinant of functional improvement in acute stroke patients: a retrospective analysis of Japan rehabilitation database. J Stroke Cerebrovasc Dis 2015;24:1019-1024. DOI:10.1016/j. jstrokecerebrovasdis.2014.12.026, PMID:25813064

42. Park RH, Allison MC, Lang J, Spence E, Morris AJ, Danesh BJ, Russell RI, Mills PR: Randomised comparison of percutaneous endoscopic gastrostomy and nasogastric tube feeding in patients with persisting neurological dysphagia. BMJ 1992;304:1406-1409. DOI:10.1136/bmj.304.6839.1406, PMID:1628013

43. Zwecker M, Levenkrohn S, Fleisig Y, Zeilig G, Ohry A, Adunsky A: Mini-Mental State Examination, cognitive FIM instrument, and the Loewenstein Occupational Therapy Cognitive Assessment: relation to functional outcome of stroke patients. Arch Phys Med Rehabil 2002;83:342-345. DOI:10.1053/apmr.2002.29641, PMID:11887114

44. Leung AW, Cheng SK, Mak AK, Leung KK, Li LS, Lee TM: Functional gain in hemorrhagic stroke patients is predicted by functional level and cognitive abilities measured at hospital admission. NeuroRehabilitation 2010;27:351-358. DOI:10.3233/NRE-2010-0619, PMID:21160125

45. Mizrahi EH, Fleissig Y, Arad M, Adunsky A: Functional gain following rehabilitation of recurrent ischemic stroke in the elderly: experience of a post-acute care rehabilitation setting. Arch Gerontol Geriatr 2015;60:108-111. DOI:10.1016/j.archger.2014.08.013, PMID:25239513

46. Arai T, Sekizawa K, Ohrui T, Fujiwara H, Yoshimi N, Matsuoka H, Sasaki H: ACE inhibitors and protection against pneumonia in elderly patients with stroke. Neurology 2005;64:573-574. DOI:10.1212/01. WNL.0000150897.14961.0F, PMID:15699404

47. Kumazawa R, Jo T, Matsui H, Fushimi K, Yasunaga H: Association between angiotensin converting enzyme inhibitors and post stroke aspiration pneumonia. J Stroke Cerebrovasc Dis 2019;28:104444. DOI:10.1016/j. jstrokecerebrovasdis.2019.104444, PMID:31635965

48. Osawa A, Maeshima S, Tanahashi N: Efficacy of cilostazol in preventing aspiration pneumonia in acute cerebral infarction. J Stroke Cerebrovasc Dis 2013;22:857-861. DOI:10.1016/j.jstrokecerebrovasdis.2012.06.008, PMID:22877690 
49. Sethi S, Murphy TF: Infection in the pathogenesis and course of chronic obstructive pulmonary disease. N Engl J Med 2008;359:2355-2365. DOI:10.1056/ NEJMra0800353, PMID:19038881

50. Yamauchi Y, Yasunaga H, Matsui H, Hasegawa W, Jo T, Takami K, Fushimi K, Nagase T: Comparison of clinical characteristics and outcomes between aspiration pneumonia and community-acquired pneumonia in patients with chronic obstructive pulmonary disease. BMC Pulm Med 2015;15:69. DOI:10.1186/s12890-0150064-5, PMID:26152178
51. Vijayan VK: Chronic obstructive pulmonary disease. Indian J Med Res 2013;137:251-269. PMID:23563369

52. Komiya K, Ishii H, Kadota J: Healthcare associated pneumonia and aspiration pneumonia. Aging Dis 2015;6:27-37. DOI:10.14336/AD.2014.0127, PMID:25657850

53. Mandell LA, Niederman MS: Aspiration pneumonia. N Engl J Med 2019;380:651-663. DOI:10.1056/NEJMra1714562, PMID:30763196 